\title{
An Ambulatory Care Clinic and Community Pharmacy Collaboration to Address Prescription Abandonment
}

\author{
Joshua W. Gaborcik, PharmD, BCPS ${ }^{1 *}$; Brigid K. Groves, PharmD, MS ${ }^{2+}$; Marilly Palettas, MPH ${ }^{3}$ Aaron D. Clark, DO ${ }^{4}$; \\ Alexa Sevin Valentino, PharmD, BCACP,5 \\ ${ }^{1}$ The Ohio State University Wexner Medical Center; ${ }^{2}$ Partners For Kids, Nationwide Children's Hospital; \\ ${ }^{3}$ The Ohio State University, Center for Biostatistics; ${ }^{4}$ The Ohio State University, College of Medicine; \\ ${ }^{5}$ The Ohio State University College of Pharmacy and PrimaryOne Health \\ *Affiliation during research: PrimaryOne Health \& The Ohio State University, College of Pharmacy \\ ${ }^{+}$Affiliation during research: The Kroger Co., Columbus Division \& The Ohio State University, College of Pharmacy \\ ${ }^{\wedge}$ Affiliation during research: PrimaryOne Health
}

\begin{abstract}
Purpose: The purpose of this research is to evaluate a collaborative workflow aimed at decreasing prescription abandonment. Setting: A federally qualified health center and a $340 B$ contracted grocery store-based community pharmacy. Practice Workflow: An ambulatory care clinic with an established partnership with a community pharmacy chain identified a need to decrease prescription abandonment rates. A process was developed whereby an ambulatory care pharmacy technician received a report from the pharmacy of prescriptions filled for at least 7 days since the initial fill date and at risk for abandonment at the community pharmacy. The pharmacy technician identified health-system barriers, attempted to remedy any identified barriers, and conducted patient reminder phone calls. Health-system barriers were classified by the following categories: incorrect contact information at the community pharmacy, incorrect 34OB copayment, incorrect insurance information at the community pharmacy, and need for prior authorization. Evaluation: A prospective cohort study was conducted from February 2016 to April 2016 in order to evaluate the effectiveness of this workflow. Results: 551 prescriptions and 350 patients were included in this cohort. Of the 551 prescriptions, 362 had at least one identified barrier that may have led to prescription abandonment. There were 111 health-system identified barriers, and 96 of these barriers were acted upon. Additionally, there were 459 patient identified barriers, and 179 of these barriers were acted upon. When a pharmacy technician was able to identify and act upon at least one barrier, 106 prescriptions (46.9\%) were picked-up from the pharmacy. Conclusion: From the information gathered in this quality improvement project, operational changes have been implemented at the ambulatory care clinic and community pharmacy as a means to further decrease modifiable health-system barriers that may lead to prescription abandonment.
\end{abstract}

Keywords: transitions of care; ambulatory care; community pharmacy; technicians; prescription abandonment

\section{Background}

In 2014, the United States healthcare expenditure reached \$3 trillion with chronic diseases accounting for $86 \%$ of healthcare costs. ${ }^{1,2}$ Prescription medications play a vital role in preventing and managing chronic diseases and associated healthcare costs. Unfortunately, patients do not always fill the prescriptions that are written for them, which negatively impacts their adherence rate. A lower medication adherence rate may lead to uncontrolled disease states, thereby increasing the likelihood of disease complications and subsequent hospital admission. . $^{3,4}$ Medication non-adherence contributes to annual hospitalization costs of $\$ 100$ billion as well as an extra $\$ 2000$ per patient in excess ambulatory care physician visits. ${ }^{3} \mathrm{~A}$ contributing factor to medication non-adherence is prescription abandonment.

Corresponding author: Alexa Sevin Valentino, PharmD, BCACP Assistant Professor of Clinical Pharmacy The Ohio State University, College of Pharmacy 500 West $12^{\text {th }}$ Avenue, Columbus, $\mathrm{OH} 43210$ Phone: (614) 292-1186; Email: valentino.49@osu.edu
Prescription abandonment occurs when a prescription has been electronically delivered, faxed, telephoned, or hand-delivered to a pharmacy, and the patient never picks up the prescription regardless of whether the prescription is a new medication or refill medication. ${ }^{5}$ Within the general public, it is estimated that prescription abandonment rates range between $3-10 \% .^{6-9}$ Common factors associated with abandonment include initiation of a new medication, electronic delivery of prescriptions, cost of the prescription, inadequate insurance coverage, lack of knowledge regarding necessity for the medication, and lack of knowledge regarding the medication. ${ }^{9-}$ 11 Additionally, studies have found that patients with chronic diseases were less likely to retrieve newly prescribed medications from their pharmacy. ${ }^{9,10}$ In order to decrease prescription abandonment rates, community pharmacies are utilizing automated voice calls, live calls, or text/email messaging to inform and remind patients that their prescriptions are available for pick-up. ${ }^{5,12,13}$ The effectiveness of these actions is unknown as there is limited literature demonstrating the effect of these interventions to prevent prescription abandonment. Additionally, there is limited literature from the clinic perspective that highlights 
interventions targeted to help prevent prescription abandonment. The purpose of this research is to evaluate a collaborative workflow aimed at decreasing prescription abandonment.

\section{Methods}

\section{Practice Settings}

PrimaryOne Health is a federally qualified health center (FQHC) recognized as a National Committee for Quality Assurance (NCQA) Tier 3 patient-centered medical home (PCMH). PrimaryOne Health has ten health centers located in socioeconomic and culturally diverse neighborhoods throughout Franklin and Pickaway counties in Ohio. Over 40,000 patients are seen within PrimaryOne Health each year. PrimaryOne Health serves all patients, including the vulnerable and un/under-insured residents of metropolitan Columbus. Services provided at PrimaryOne Health include adult medicine, preventative care, pediatrics, OB-GYN, clinical pharmacy support, nutrition, and behavioral health, as well as dental and vision services. The pharmacy department provides medication therapy management for diabetes, hypertension, dyslipidemia, COPD, and smoking cessation. At the time of this evaluation, the pharmacy department consisted of a shared clinical faculty member, one-part time pharmacist, a post-graduate year 2 pharmacy resident, and two pharmacy technicians. Although PrimaryOne Health provides comprehensive care, including clinical pharmacy services, it does not have an on-site pharmacy.

In 2007, The Kroger Co., Columbus Division became a contracted 340B pharmacy for the FQHC. The Kroger Co., Columbus Division is comprised of 120 grocery-store based community pharmacies across the states of Ohio, West Virginia, and Michigan. At the time of this evaluation, eight of these 120 pharmacies, located in the greater central Ohio area, were contracted 340B pharmacies that collaborate with PrimaryOne Health to provide prescriptions and pharmacy services to eligible patients. Services provided by Kroger Pharmacy include medication therapy management, immunization assessment and administration, point-of-care testing, and medication synchronization among others. The eight contracted 340B Kroger Pharmacies are strategically located near a PrimaryOne Health clinic in order to optimize the care of PrimaryOne Health patients.

\section{Practice Workflow}

With the charge from national pharmacy organizations to consider care transitions and a mission of forward-thinking pharmacy practice models ${ }^{14-16}$, the pharmacy staff at PrimaryOne Health and Kroger Pharmacy collaborated to design a process aimed to decrease the number of abandoned prescriptions at Kroger Pharmacy, and ensure that patients cared for in the community receive the medications necessary for chronic disease management.
At PrimaryOne Health, one of the pharmacy technicians serves as the 340B Coordinator, who is responsible for the day-to-day management of the PrimaryOne Health 340B network. In an attempt to identify reasons for high prescription abandonment among PrimaryOne Health patients and to decrease prescription abandonment, Kroger Pharmacy provided the PrimaryOne Health 340B Coordinator a twice weekly report of prescriptions at risk for abandonment written by providers within the PrimaryOne Health network. Prescriptions at risk for abandonment are defined as an active prescription that has been filled, has been waiting to be picked-up for at least seven days since fill date, and has less than or equal to seven days before being returned to stock. Prescriptions filled at Kroger Pharmacy have 14 days from the initial fill date to be picked up by the patient otherwise the prescription is returned to stock and is considered abandoned. Each line of the report was unique to a specific patient and contained the patient name, date of birth, medical record number, contact information on file at Kroger Pharmacy, prescription name, generated prescription copay based on $340 \mathrm{~B}$ coverage or insurance information at the community pharmacy, and the dispensing pharmacy address. The 340B Coordinator analyzed the report for barriers, corrected any identified issues, followed-up with the Kroger or PrimaryOne Health pharmacist and/or healthcare provider as needed, and called patients to remind them prescriptions were available for pick-up. The process is outlined in Figure 1. This process started in March 2015, and continued until September 2016.

\section{Evaluation}

In order to evaluate the impact of this practice innovation, PrimaryOne Health in collaboration with Kroger Pharmacy and The Ohio State University College of Pharmacy collected data from February 2016 to April 2016. The primary objective was to describe and summarize observed barriers to picking up prescriptions from a community pharmacy. Secondary objectives were to classify types of abandoned prescriptions, report the number of prescriptions picked-up after the pharmacy technician intervention, and quantify amount of time per week a pharmacy technician spends assisting with abandoned prescriptions. The Ohio State University Institutional Review Board approved the study.

Patients were eligible for inclusion in the study if they: 1) established care within a PrimaryOne Health clinic; 2) had active medications at PrimaryOne Health being filled at a contracted 340B Kroger pharmacy; 3) had at least one prescription at risk of being abandoned; and 4) were 18 years of age or older. Prescriptions were excluded from the study if they were filled at a community pharmacy other than the contracted Kroger 340B pharmacies or were filled through a manufacturer patient assistance program. Data were obtained from the report provided by Kroger Pharmacy and PrimaryOne Health electronic health record. Data were collected at the patient-level and prescription-level. Patient-level data included: age, gender, race, ethnicity, primary spoken 
language, employment status, marital status, healthcare center, date of service, insurance status, total number of prescriptions at risk of being abandoned, and number of new and refill prescriptions at risk of being abandoned. Prescriptionlevel data included: barriers to picking-up prescriptions, correction of barrier, medication class, dosage form of medication, whether the prescription is a new medication for chronic disease, and whether the prescription was picked-up from pharmacy after intervention.

Barriers to picking-up prescriptions were divided into healthsystem and patient identified barriers. A prescription could have more than one barrier identified. Health-system identified barriers were classified as follows: incorrect contact information at the community pharmacy, incorrect 340B copayment, incorrect insurance information at the community pharmacy, and need for prior authorization. Patient identified barriers were classified as follows: lack of awareness/knowledge of the medication, primary spoken language different than English, inability to pay for medication despite correct copay, lack of transportation to the pharmacy, and other. Patient identified barriers were recorded if the patient voluntarily provided the information during the reminder phone call conducted by the pharmacy technician, except language. Language was recorded as a barrier if an interpreter was needed to conduct the patient reminder calls. During study design, it was known that certain patient identified barriers - language, transportation, and cost of medication - could not be acted upon by the 340B Coordinator; however, it was important for PrimaryOne Health and Kroger Pharmacy to know how often these barriers arose in order to consider process improvement solutions. For the health-system barriers, the pharmacy technician acted upon the barrier by supplying the community pharmacy with the appropriate information, and consulting with providers and clinical pharmacists as needed. For patient identified barriers, the pharmacy technician acted upon the barrier, if possible. Every patient, regardless of whether a barrier was identified or not, was provided a reminder call that the prescription was ready for pick-up.

\section{Statistical analysis}

Descriptive statistics were used to summarize all data items including how frequently certain barriers were observed, how long the pharmacy technician spent correcting barriers and the types of prescriptions abandoned. Data items were summarized at the patient level or prescription level. Continuous variables were expressed using means, medians, standard deviations and other appropriate measures of spread and categorical variables using frequencies and percentages. A chi-squared test was used to test if among those prescriptions that had a barrier, was there a difference in proportion of prescriptions picked-up between those who had any barrier corrected compared to those with no barriers corrected. All analyses were performed using SAS version 9.4, SAS Institute, Cary, North Carolina.

\section{Results}

A total of 557 prescriptions were at risk for abandonment at the community pharmacies, and 551 prescriptions were included in data analysis. Six prescriptions were excluded as the prescriptions were not filled at Kroger Pharmacy. The 551 prescriptions were prescribed to 350 unique individuals. Baseline demographics for these patients are summarized in Table 1. Overall, the majority of patients were female (66\%) with an average age of 47 . The majority of the patient cohort was Hispanic (53.7\%) followed by African American (25.7\%) and Caucasian (18.3\%). The primary spoken language for the majority of patients was Spanish (57.7\%) followed by English $(40.6 \%)$. Finally, the majority of patients were uninsured (83.4\%).

Table 2 describes identified and resolved barriers to picking up prescriptions. Within the total cohort of 551 prescriptions, 362 prescriptions had at least one barrier identified. Of the 362 prescriptions, there were 111 health-system barriers identified, and the pharmacy technician acted upon 96 of these barriers. The most common health-system barrier was incorrect contact information at the community pharmacy, and the pharmacy technician was able to correct this barrier $93.2 \%$ of the time.

Of the 362 prescriptions, there were 459 patient barriers identified and of those, the pharmacy technician acted upon 179 barriers. The most common patient barrier was a lack of awareness or knowledge that the medication was ready at the community pharmacy. Language (178 instances), cost (32 instances), and transportation barriers (13 instances) account for 223 of the 459 patient barriers identified; however, the pharmacy technician did not act upon these barriers. Within the "other" category, the most common barrier was inability to reach the patient (48 instances) followed by patient refusal of the medication (6 instances).

Of the 362 prescriptions with at least one barrier identified, there were 226 prescriptions that had at least one barrier acted upon; within this cohort, 106 (46.9\%; p-value 0.0804) prescriptions were picked-up from the pharmacy. Similarly, 94 prescriptions had multiple barriers and multiple interventions by the pharmacy technician with $42(44.7 \%)$ of the prescriptions picked-up from the pharmacy. Of the 362 prescriptions with at least one barrier identified, 157 (43.4\%) were picked up from the pharmacy either before or without the barrier being acted upon. Prescription demographics are summarized in Table 3.

Of the 551 prescriptions at risk of being abandoned at the community pharmacy, $458(83.1 \%)$ prescriptions were refill medications used to treat chronic diseases including hypertension (22.1\%), diabetes (20.5\%), and hyperlipidemia (9.8\%). The pharmacy technician spent an average of 3.8 hours per report per week. Kroger Pharmacy sent two reports per week; however, despite identifying barriers, correcting barriers, and reminding patients that prescriptions are ready for pick-up, 
a majority of prescriptions were abandoned (54.4\%) at the community pharmacy.

\section{Discussion}

Through this clinic and community pharmacy collaboration, we identified several common barriers that may be impacting prescription abandonment. While the intervention by the ambulatory care pharmacy technician did not have a statistically significant effect on decreasing prescription abandonment rates, we did learn useful information that led to other workflow changes to address some of the most common barriers.

Patient identified barriers occurred more frequently than health-system barriers, but the pharmacy technician was less likely to be able to act upon these barriers. For instance, the pharmacy technician does not possess the resources to coordinate or provide transportation to and from the community pharmacy. The 340B Kroger Pharmacies provide prescription home delivery at no charge, but patients must live within 10 miles of the store. Education about this service was provided to the clinic providers so they could educate patients that may benefit from this service.

When it comes to language, the 340B pharmacy technician has access to interpreters for non-English speaking patients, and these interpreters are utilized when making patient reminder calls. However, it is up to the patient to navigate the process of getting to the pharmacy and communicating with clinical support staff and pharmacists at the community pharmacy in order to obtain their prescriptions. Kroger Pharmacy does have interpreter services in the contracted $340 \mathrm{~B}$ pharmacies; however, utilization of this service was not examined in this study. Similar to the previous barrier, further education and advertisement of interpreter services was provided to the clinic providers and staff so that they could educate patients that might benefit from that service.

The results of this evaluation support previously identified reasons for prescription abandonment, while at the same time highlighting additional reasons why prescriptions may never be picked up from the community pharmacy. Our evaluation confirms that patients are less likely to pick-up prescriptions if patient identified barriers include cost of a medication, and/or lack of knowledge or awareness of the medication availability or its necessity. ${ }^{9-11}$ Reasons for abandoning prescriptions are complex and may include one or more of the following explanations: financial considerations; poor health literacy; feasible access to the pharmacy; and more. ${ }^{9-11,17-21}$ While research has identified reasons for not picking up a prescription, effective solutions are needed to improve prescription abandonment rates.

Based on the time required for the pharmacy technician to complete one report and the lack of statistically significant impact, the PrimaryOne Health pharmacy technician was removed from this workflow. However, several operational process changes were implemented based upon the findings of this study. PrimaryOne Health implemented an internal process to ensure: a) correct contact information is updated within the electronic health record and thus submitted to the community pharmacy on the prescription: and b) patient is assigned to the correct 340B copay bracket to ensure patients' are charged the appropriate copay. Kroger Pharmacy implemented an internal process to verify a patient's personal information on the prescription matches the contact information in Kroger Pharmacy's data entry software. This helps to ensure that patients receive reminders when they have medications that are ready to be picked up.

Limitations to the practice innovation include personnel involved in the practice workflow. Kroger identified one individual who was responsible for running the reports supplied by Kroger Pharmacy, and PrimaryOne Health had one individual tasked with analyzing the reports. If one of these individuals was not able to perform their respective duties, then the at-risk prescription abandonment reports were not created nor analyzed, barriers were not corrected nor acted upon, and patients did not receive a reminder phone call from the PrimaryOne Health pharmacy technician. During the 12-week study period, there were three weeks when reports were not completed due to the absence of key personnel from PrimaryOne Health and/or Kroger Pharmacy. Also, due to the time-sensitivity of the reports and volume of patients that appear on one report, it can be inefficient to work on outdated reports. During the study period, the PrimaryOne Health Pharmacy Department experienced some internal changes including loss of an additional pharmacy technician. This change resulted in more responsibility being placed on the pharmacy technician who is responsible for working on the Kroger supplied reports, as well as relocation of the pharmacy technician, whereby interpreter access was limited.

Additionally, the clinic and community pharmacy did not share an integrated health record and dispensing software. As a result, there are a number of confounding variables when evaluating this practice innovation. First, auto-refill was not assessed during this study. Active prescriptions from PrimaryOne Health were included in the study; however, there was no process to assess if an inactive prescription was still on auto-refill at the community pharmacy. Secondly, medicationpossession ratio was not assessed during this study, which could explain why refill medications were more likely to be abandoned than new prescriptions.

There are also limitations to the results of this evaluation including the non-randomized, single-center study design. The single-center study design including only PrimaryOne Health and Kroger Pharmacy limits generalizability as patient demographics may vary across other collaborations between health-systems, ambulatory care clinics, and community pharmacies. Additionally, the single-center study design and 
limiting time period of the intervention contribute to the inability to determine if the ambulatory care technician made significant impact on prescription abandonment.

Future research opportunities include prospective evaluation of the health-system identified barriers from Kroger Pharmacy perspective, as well as studying the effect of decreasing prescription abandonment rates on chronic disease outcomes. Future research should account for auto-refill and medication possession ratio. Moreover, research is needed to understand patient perceptions of their chronic disease and the necessity of chronic medications in diverse cultural and socioeconomic patient populations. This may help to identify a more targeted intervention to decrease prescription abandonment rates for refill medications.

\section{Conclusion}

As healthcare costs in the United States continue to rise, targeted interventions are needed to decrease prescription abandonment rates, and enable patients to obtain their chronic disease medications. For PrimaryOne Health and The Kroger Co., Columbus Division it is a priority to provide optimal care to our culturally diverse patient populations, and in working towards this mission, prescription abandonment became a focus. Although our process, whereby an ambulatory care pharmacy technician identified and corrected barriers that may have led to prescription abandonment, is no longer being utilized, internal operation improvements at PrimaryOne Health and Kroger Pharmacy have been implemented to help decrease prescription abandonment. By highlighting this process, health-systems, ambulatory care clinics, and community pharmacies are encouraged to identify specific barriers that may lead to prescription abandonment in their unique settings in order to design a process that helps improve transitions of care for patients.

\section{Funding: None}

Conflicts of Interest: None

Acknowledgements: The authors would like to thank Clinton Scott, CPhT, Emily Riley, PharmD, and Allison Wehr, MS for their contributions to this project.

Treatment of Human Subjects: IRB review/approval required and obtained

\section{References}

1. National Health Expenditure Data - Historical 2015. http://www.cms.gov/research-statistics-data-andsystems/statistics-trends-andreports/nationalhealthexpenddata/nationalhealthaccounts historical.html. Accessed June 11, 2016.

2. Centers for Disease Control and Prevention. Chronic Disease Prevention and Health Promotion 2016. http://www.cdc.gov/chronicdiseae/. Accessed June 11, 2016.

3. Osterberg L, Blaschke T. Adherence to medication. N Engl J Med. 2005;353(5):487-497.
4. Chisholm-Burns MA, Spivey CA. The 'cost' of medication nonadherence: consequences we cannot afford to accept. J Am Pharm Assoc. 2012;52(6):823-826.

5. Hubbard TE. Ready for pick-up: reducing primary medication non-adherence. The Network for Excellence in Health Innovation.

http://www.nehi.net/writable/publication_files/file/pmn_is sue_brief_10_14_formatted_final.pdf. Accessed June 11, 2016.

6. Aitken $M$, Kleinrock $M$, Lyle J, et al. Medicine use and shifting costs of healthcare, a review of the use of medicines in the United States in 2013. IMS Institute for Healthcare Informatics.

http://www.plannedparenthoodadvocate.org/2014/IIHI_US _Use_of_Meds_for_2013.pdf. Accessed December 13, 2016.

7. Pace $P$, Holmes $E$, West-Strum D. Store and prescription characteristics associated with primary medication nonadherence. J Manag Care Spec Pharm. 2014;20(8):824832.

8. Raebel MA, Ellis JL, Carrol NM, et al. Characteristics of patients with primary non-adherence to medications for hypertension, diabetes, and lipid disorders. J Gen Intern Med. 2012;27(1):57-64.

9. Shrank WH, Choudhry NK, Fischer MA, et al. The epidemiology of prescriptions abandoned at the pharmacy. Ann Intern Med. 2010;153(10):633-640.

10. Fischer MA, Stedman MR, Lii J, et al. Primary medication non-adherence: analysis of 195,930 electronic prescriptions. J Gen Intern Med. 2010;25(4):284-290.

11. Kennedy J, Tuleu I, Mackay K. Unfilled prescriptions of Medicare beneficiaries: prevalence, reasons, types of medicines prescribed. J Manag Care Pharm. 2008;14(6):553-560.

12. Fischer MA, Choudhry NK, Bykov K, et al. Pharmacy-based interventions to reduce primary medication nonadherence to cardiovascular medications. Med Care. 2014;52(12):1050-1054.

13. Xie J, Gallagher L, Wurst AM, Shrank WH. A randomized telephone intervention trial to reduce primary medication nonadherence. J Manag Care Pharm. 2015;21(2):124-131.

14. Introduction to the proceedings of the summit. Am J Health Syst Pharm. 2014;71(16):1345-1347.

15. Recommendations of the summit. Am J Health Syst Pharm. 2014;71(16):1390-1391.

16. Hume AL, Kirwin J, Bieber HL, et al. Improving care transitions: current practice and future opportunities for pharmacists. Pharmacotherapy. 2012;32(11):e326-337.

17. Mullins CD, Blatt L, Gbarayor CM, et al. Health disparities: a barrier to high-quality care. Am J Health Syst Pharm. 2005;62(18):1873-1882.

18. Weiss BD, Hart G, Pust R. The relationship between health literacy and health. J Health Care Poor Underserved. 1991;1:351-363.

19. Tkacz VL, Metzger A, Pruchnicki MC. Health literacy in pharmacy. Am J Health Syst Pharm. 2008;65(10):974-981.

20. National Center for Education Statistics. 2006. The Health Literacy of America's Adults: Results From the 2003 National Assessment of Adult Literacy. Washington, DC: U.S. Department of Education.

21. Heins JR. Prescription abandonment: causes and prevention. S D Med. 2011;64(2):51-53. 
Figure 1 - The Process
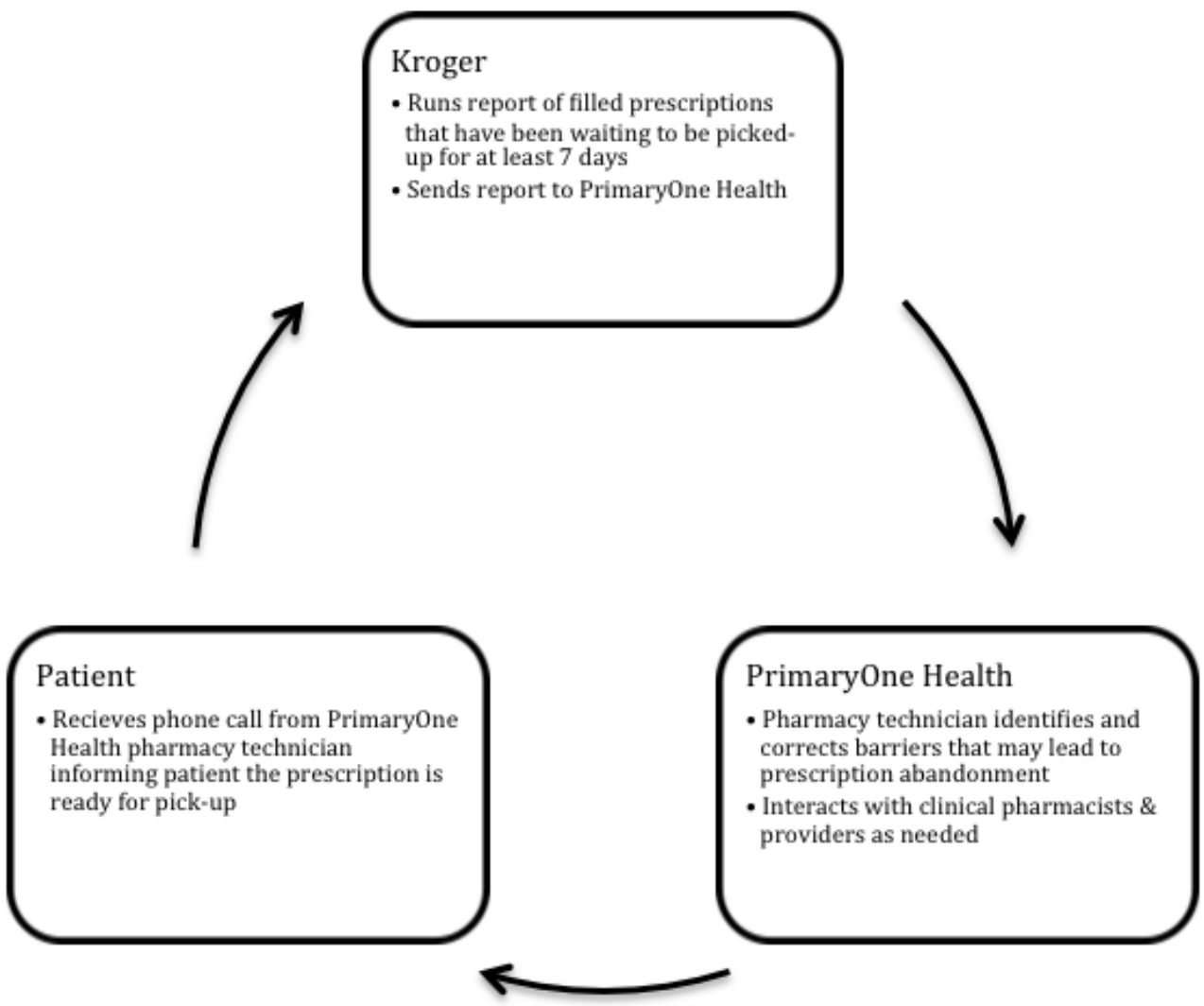
Table 1 - Baseline Demographics

\begin{tabular}{|lr|}
\hline & Total $(n=350)$ \\
\hline Age [Mean (SD)] & $47.8(11.9)$ \\
\hline Sex [n (\%)] & \\
Female & $231(66)$ \\
Male & $119(34)$ \\
\hline Race $[n$ (\%)] & \\
Not reported & $188(53.7)$ \\
African American & $90(25.7)$ \\
Caucasian & $64(18.3)$ \\
Asian & $6(1.7)$ \\
Native American & $2(0.6)$ \\
\hline Ethnicity [n (\%)] & \\
Hispanic or Latino & $209(59.7)$ \\
Non-Hispanic & $141(40.3)$ \\
\hline Primary Language [n (\%)] & \\
Spanish & $202(57.7)$ \\
English & $142(40.6)$ \\
Other & $4(1.1)$ \\
Arabic & $1(0.3)$ \\
Vietnamese & $1(0.3)$ \\
\hline Employment [n (\%)] & \\
Unemployed & $142(40.6)$ \\
Full time & $129(36.9)$ \\
Part time & $72(20.6)$ \\
Undefined & $6(1.7)$ \\
Retired & $1(0.3)$ \\
\hline Marital Status [n (\%)] & \\
Single & $176(50.3)$ \\
Married & $123(35.1)$ \\
Divorced & $39(11.1)$ \\
Widowed & $12(3.4)$ \\
\hline Insurance Status $[n(\%)]$ & \\
Uninsured & $292(83.4)$ \\
Medicaid & $14(4)$ \\
Medicare & $2(0.6)$ \\
Private Insurance & \\
\hline & Antar \\
\hline
\end{tabular}

${ }^{*}$ Amharic (2), Fulah (1), unknown (1) 
Table 2 - Barriers to picking up prescriptions

\begin{tabular}{|c|c|c|c|}
\hline Prescriptions at risk for abandonment at 340B pharmacy, $n$ & & 551 & \\
\hline Prescriptions with no identified barriers, $n$ & & 189 & \\
\hline Prescriptions with at least one barrier identified, $\mathrm{n}$ & & 362 & \\
\hline \multirow[t]{2}{*}{ Prescriptions with more than one barrier identified, $\mathrm{n}$} & & 171 & \\
\hline & $\begin{array}{c}\text { Barrier } \\
\text { Identified } \\
\mathrm{n}(\% \text { of total at } \\
\text { risk) }\end{array}$ & $\begin{array}{c}\text { Barrier } \\
\text { Acted Upon } \\
\mathrm{n} \text { (\% of barrier } \\
\text { identified) } \\
\end{array}$ & $\begin{array}{l}\text { Prescription } \\
\text { Picked-up } \\
\mathrm{n} \text { (\% of barrier } \\
\text { identified) } \\
\end{array}$ \\
\hline \multicolumn{4}{|l|}{ Health-System Barriers } \\
\hline Incorrect contact information for patient & $44(8.0)$ & $41(93.2)$ & $25(56.8)$ \\
\hline Incorrect 340B copayment & $33(6.0)$ & $25(75.8)$ & $12(36.4)$ \\
\hline Incorrect insurance information & $23(4.2)$ & $19(82.6)$ & $9(39.1)$ \\
\hline Prior authorization needed & $11(2.0)$ & $11(100.0)$ & $4(36.4)$ \\
\hline TOTAL & $111(20.1)$ & $96(86.5)$ & $50(45.0)$ \\
\hline \multicolumn{4}{|l|}{ Patient-related Barriers } \\
\hline Awareness/Knowledge of the medication & $180(32.7)$ & $176(97.8)$ & $89(49.4)$ \\
\hline Language & $178(32.3)$ & $0(0.0)$ & $88(49.4)$ \\
\hline Cost of medication & $32(5.8)$ & $0(0.0)$ & $8(25.0)$ \\
\hline Transportation & $13(2.3)$ & $0(0.0)$ & 7 (53.9) \\
\hline Other* & $56(10.2)$ & $3(0.5)$ & $14(25.0)$ \\
\hline TOTAL & 459 (83.3) & $179(39.0)$ & 206 (44.9) \\
\hline
\end{tabular}

*Other barriers include: inability to reach patient (48), patient refusal of medication (6), coordination of care with case manager (1), and insurance coverage (1).

Table 3 - Prescription Demographics

\begin{tabular}{|lr|}
\hline Prescriptions at risk of abandonment at 340B pharmacy, $\mathrm{n}$ & 551 \\
Prescriptions picked up from pharmacy with no barriers corrected, $\mathrm{n}$ & 157 \\
Prescriptions picked up from pharmacy with at least one barrier corrected, $\mathrm{n}^{\wedge}$ & 106 \\
Prescriptions picked up from pharmacy with multiple barriers corrected, $\mathrm{n}^{\wedge}$ & 42 \\
\hline Medications classified by disease [n (\%)] & $122(22.1)$ \\
Hypertension & $113(20.5)$ \\
Diabetes & $54(9.8)$ \\
Hyperlipidemia & $38(6.9)$ \\
Pulmonary & $26(4.7)$ \\
Depression & $2(0.4)$ \\
Smoking cessation & $195(34.7)$ \\
Other & \\
\hline
\end{tabular}

${ }^{\wedge}$ A prescription can be included in more than category

${ }^{*}$ Other medications treated the following conditions: Pain medications (NSAIDS plus gabapentin) (46), Gastroesophageal reflux disease (36), Women's Health (18), Allergies + Nasal Congestion (17), Endocrinology disorders (16), Infectious diseases (15), Genitourinary disorders (7), Topical medications (6), Corticosteroids (6), Anti-epileptics (2), and miscellaneous (26). One 'other' medication was not recorded by class of medication so the total 'medications classified by disease' is 550 prescriptions. 